(C) 1997 Cambridge University Press

\title{
Numerical simulation of dynamic contact-line problems
}

\author{
By IVAN B. BAZHLEKOV† AND PETER J. SHOPOV \\ Institute of Mathematics, Bulgarian Academy of Sciences, acad. G. Bonchev str., bl. 8, \\ 1112 Sofia, Bulgaria
}

(Received 10 June 1993 and in revised form 18 June 1997)

The presence of a three-phase region, where three immiscible phases are in mutual contact, causes additional difficulties in the investigation of many fluid mechanical problems. To surmount these difficulties some assumptions or specific hydrodynamic models have been used in the contact region (inner region). In the present paper an approach to the numerical solution of dynamic contact-line problems in the outer region is described. The influence of the inner region upon the outer one is taken into account by means of a solution of the integral mass and momentum conservation equations there. Both liquid-fluid-liquid and liquid-fluid-solid dynamic contact lines are considered. To support the consistency of this approach tests and comparisons with a number of experimental results are performed by means of finite-element numerical simulations.

\section{Introduction}

Dynamic contact-line problems arise in the study of many processes such as injection moulding, vaporization of volatile liquid drops in an immiscible less-volatile liquid, spreading of adhesives, coating of solid surfaces with a thin liquid layer, etc. Although much has been done recently to solve these problems (see e.g. reviews by Dussan V. 1979 and de Gennes 1985), a final solution is still not known. A full analysis of many such problems is inhibited by the inability to find an appropriate model of the contact region and the contact line.

Depending on the phases which are in mutual contact there are two main contactline types: liquid-fluid-liquid ( $\mathrm{L}-\mathrm{F}-\mathrm{L})$ and liquid-fluid-solid $(\mathrm{L}-\mathrm{F}-\mathrm{S})$. In the static case the boundary conditions at the contact line are

the Antonow rule in the $\mathrm{L}-\mathrm{F}-\mathrm{L}$ case:

$$
\left.\begin{array}{c}
\sigma_{23} \sin \theta_{2}-\sigma_{13} \sin \theta_{1}=0 \\
\sigma_{12}+\sigma_{23} \cos \theta_{2}+\sigma_{13} \cos \theta_{1}=0,
\end{array}\right\}
$$

where $\sigma_{i j}$ is the surface tension coefficient at the interface $\Gamma_{i j}, \theta_{i}$ are the static contact angles (see figure 1); and subscripts 1, 2, and 3 refer to the liquid, fluid, and liquid regions respectively;

the Young equation in the $\mathrm{L}-\mathrm{F}-\mathrm{S}$ case:

$$
\sigma_{13}-\sigma_{23}=-\sigma_{12} \cos \theta_{s}
$$

where $\theta_{s}$ is the static contact angle and subscripts 1,2 and 3 refer to the liquid, fluid and solid regions respectively. There are, however, different interpretations of the static

$\dagger$ Present address: Faculty of Mechanical Engineering, TU Eindhoven, 5600 MB Eindhoven, The Netherlands; e-mail:bazhleko@wfw.wtb.tue.nl. 
(a)

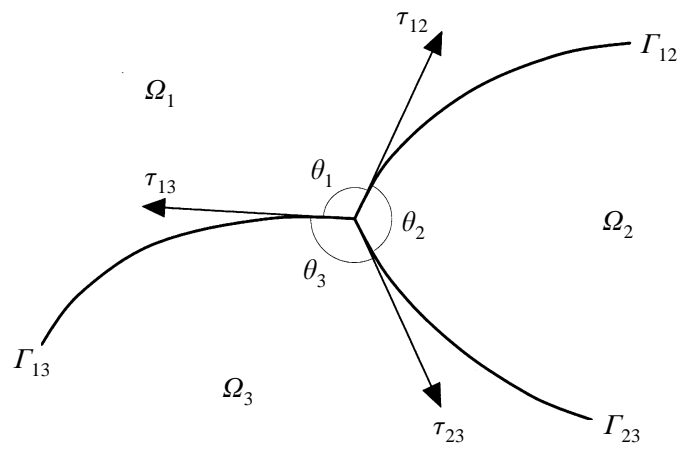

(b)

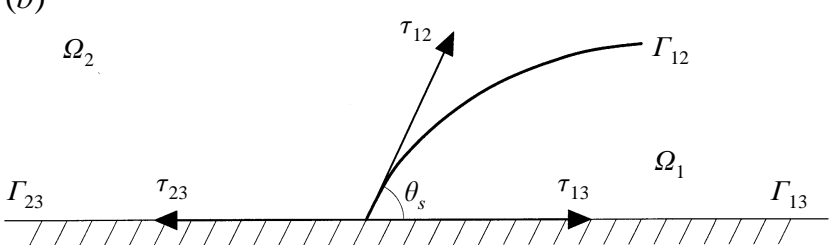

FigURE 1. Schematic diagram of the static contact-line vicinity: (a) a liquid-fluid-liquid contact line, $(b)$ a liquid-fluid-solid contact line.

conditions (1.1) and (1.2): some authors (e.g. Batchelor 1967 and Johnson \& Sadhal 1985) have assumed that the $\sigma_{i j}$ are known up to the contact line and have considered (1.1) and (1.2) as a force balance at this line; others (e.g. Dussan V. 1979, de Gennes 1985 and Sullivan \& Telo da Gama 1986) have pointed out that the parameter $\sigma_{i j}$ describes adequately the energy of the interface $\Gamma_{i j}$ outside a vicinity of the contact line and equations (1.1) and (1.2) relate apparent contact angles $\theta_{k}$ to the far-field energies $\left(\sigma_{i j}\right)$. In the contact-line vicinity the structure is much more complex and its influence can be taken into account by adding 'line tension', e.g. like Pethica (1977) and Ivanov, Kralchevsky \& Nikolov (1986), or by considering intermolecular forces, like Hocking (1993).

In the case of dynamic contact-line problems there are two general questions which have been under intensive investigation recently, but no reliable answers exist yet. The first question, which seems to occur only in the case of a moving $\mathrm{L}-\mathrm{F}-\mathrm{S}$ contact line and it is related to the force singularity at the contact line, arises when the Navier-Stokes equations and the no-slip boundary condition are used in the whole fluid region and on the whole solid surface. Various approaches to remove this singularity exist (see reviews by Dussan V. 1979 and de Gennes 1985). In the present paper it is removed by applying a slip boundary condition at the solid surface in the contact-line vicinity (see $\S 5$ ). However, our attention is not directed at the identification of the appropriate removal technique of this singularity, and, because of that, this question is not discussed in detail here.

The second question concerns the identification of an appropriate boundary condition at the contact line, and it arises in both $\mathrm{L}-\mathrm{F}-\mathrm{S}$ and $\mathrm{L}-\mathrm{F}-\mathrm{L}$ dynamic cases. The main attention in the literature is concentrated on the $\mathrm{L}-\mathrm{F}-\mathrm{S}$ case due to its apparent importance and wide applicability. The serious theoretical and experimental difficulties observed in the $\mathrm{L}-\mathrm{F}-\mathrm{L}$ case explain why so little has been done on it, as it is apparently more complicated due to the greater number of fluids involved.

In the majority of the theoretical investigations on problems containing a dynamic 
contact line, contact-angle values have been used as a boundary condition for the equations of the fluid-liquid interface shape. Depending on the region where the problems have been solved and the position on the free interface where its slope has been prescribed these investigations can be divided into two groups.

(i) In the first group the problem has been solved in the whole liquid region. Studies have been based on the assumption that the Navier-Stokes equations and the boundary condition at the liquid-fluid interface are valid up to the contact line and thereby the value of the contact angle $\theta^{\circ}$ has been used as a boundary condition at the contact line (Dussan V. 1976; Hocking 1977; Huh \& Mason 1977; Hocking \& Rivers 1982, etc.). Depending on the value of $\theta^{\circ}$ this group can be divided into two main subgroups: $(a)$ the dynamic contact angle is equal to the static one; $(b)$ the dynamic contact angle is a known function of the contact-line velocity. Hypotheses $(a)$ and $(b)$ have been discussed by Hocking (1992) and he has given arguments in favour of $(a)$.

(ii) In the second group the problem is solved in the outer region, where an appropriate model of the fluid motion is known. Based upon results of the existing analyses of fluid flows containing an L-F-S contact line, Kafka \& Dussan V. (1979) and Ngan \& Dussan V. (1989) have pointed out the general idea that the motion of the fluids in the outer region can be completely determined in terms of only one experimentally measureable parameter, which is independent of the overall geometry of the outer region, and it serves as a boundary condition for the equations in this region. As a such parameter they have introduced $\theta^{R}$-the inclination of the free surface measured at a specified distance $R$ from the contact line. They have suggested that an explicit model of the dynamics in the inner region may not be required for determining the movement of fluids in the outer region. Dussan V., Rame \& Garoff (1991) have experimentally confirmed this hypothesis for small capillary numbers $\left(C a<8.3 \times 10^{-3}\right)$. But all theoretical and experimental investigations in this direction have been performed under the restriction of small capillary number and negligible Reynolds number and this approach is perhaps applicable only for these cases. For instance at $C a=8.7 \times 10^{-3}$ Dussan V. et al. (1991) have obtained a systematic deviation between experiment and theory.

In the L-F-L case the experiments of Torza \& Mason (1970) and Mori (1978) clearly demonstrate that the dynamic contact angles are not constant and depend on the flow. Torza \& Mason (1970) have studied three-phase interactions in shear and electrical fields. In their table 1, systems 6 and 20, they give data for three-phase systems for which equation (1.1) can never be satisfied (one of the surface tension coefficients $\sigma_{i j}$ is greater than the sum of the other two). In the absence of external forces such systems do not have a static state in a configuration containing contact line, but in dynamic cases the contact line can exist and in some of them the actual contact angles can take any value in the interval $[0 ; \pi]$. So in this case the dynamic contact angles depend on the flow and the actual dynamic contact angles cannot always be equal to the static ones.

For $\mathrm{L}-\mathrm{F}-\mathrm{S}$ systems this situation,

$$
\left|\sigma_{13}-\sigma_{23}\right|>\sigma_{12},
$$

has been discussed by de Gennes (1985), i.e. equation (1.2) can never be satisfied and consequently in these cases the static contact angle cannot be defined by Young's equation.

As far as we know, data for an explicit contact angle dependence on the flow structure are not available for the $\mathrm{L}-\mathrm{F}-\mathrm{L}$ case, and because of that hypothesis $(\mathrm{i}, a)$ has usually been applied (e.g. Johnson \& Sadhal 1983 and Vuong \& Sadhal 1989). 
To summarize, it is clear that the majority of investigators have employed the general concept that the dynamic contact angles should be known and they can be used as a boundary condition at the contact line or to close the mathematical model in the outer region.

In the present work an approach to numerical simulation of dynamic contact-line problems in the outer region is proposed. It is based on mass and momentum conservation in a contact line vicinity: we look for a solution in the outer region, which matches the integral mass and momentum conservation equations in the inner region. This approach is described in $\S 2$. Sections 3 and 4 are devoted to the outer and inner regions respectively. In $\S 5$ the mathematical formulation of the problem is described and the correctness of the approach is discussed. Section 6 contains a brief description of the numerical technique and tests. To support the consistency of this approach comparisons with experimental results are presented in $\S 7$.

\section{The approach}

The approach described in the present paper for the numerical simulation of dynamic contact-line problems is based on some of the ideas of previous studies (see e.g. Kafka \& Dussan V. 1979 and Ngan \& Dussan V. 1989). Two regions are considered: an outer region $\widetilde{\Omega}^{r}$, where the appropriate model is known, and an inner region $\Omega^{r}$, a contact line vicinity. In the above-mentioned papers it has been pointed out that the solution in the outer region can be completely determined in terms of one experimentally measureable parameter - the inclination $\theta^{R}$ of the free surface at some distance $R$ from the solid surface. Using such a parameter the influence of the inner region on the outer one can be taken into account without solving the problem in the inner region. The paper of Boender, Chesters \& van der Zanden (1991) can also be related to this approach.

In the present paper it is assumed that in the inner region there is a very small contact line vicinity, a 'core', where an appropriate model describing the dynamic behaviour of the fluids is not known and consequently it is impossible to obtain a reliable detailed solution there. In the residual part of the inner region $\dagger$ we assume that there is an appropriate model describing the fluid dynamics; however, a detailed solution cannot be obtained in this part of the inner region because the dynamic influence of the "core' on it is unknown. Thus the main assumption in this paper is that the fluids in the 'core' act directly on the residual part of the inner region, but their influence on the outer region is negligible. In other words if a mathematical model is valid in most of the inner region, then it could be possible using an integral solution of this model in the whole inner region to take into account its influence on the outer one. In this way, a solution in the outer region could be obtained without detailed knowledge of the solution in the inner one.

This approach has some advantages compared with the existing ones: experimental data for the apparent contact angles (slope of the interfaces at a specific distance from the contact line) are not required; and it does not need detailed knowledge of the solution in the inner region (in particular the values of the actual contact angles). However, this approach has two principal drawbacks. First, detailed information on the dynamic behaviour of the fluids in the inner region and the values of the actual contact angles cannot be obtained (such information could be obtained only if the appropriate model in the inner region were known). Secondly, in the case of a moving

$\dagger$ Of course, strictly speaking the real situation is much more complicated and such regions ('core', inner and outer) can not be exactly specified. 
L-F-S contact line a slip boundary condition on the solid surface is applied to remove the force singularity, so in this case the results depend on a parameter (slip coefficient/length). In the case of a $\mathrm{L}-\mathrm{F}-\mathrm{L}$ contact line, however, the standard parameters of the three-phase system (viscosities, densities, surface tension coefficients and the geometrical parameters) are sufficient to solve the problem. Because of that, in $\S 7$ comparisons with experimental data are presented mainly for $\mathrm{L}-\mathrm{F}-\mathrm{L}$ contact-line problems.

\section{The outer region}

In this section the mathematical model in the outer region $\tilde{\Omega}^{r}=\Omega \backslash \Omega^{r}\left(\Omega^{r}\right.$ is the inner region) is formulated. It includes the equations of motion of the $i$ th fluid in the outer region $\widetilde{\Omega}_{i}^{r}=\widetilde{\Omega}^{r} \cap \Omega_{i}$ :

conservation of mass

$$
\boldsymbol{\nabla} \cdot \tilde{\boldsymbol{v}}^{(i)}=0
$$

conservation of momentum

$$
\rho_{i}\left(\frac{\partial \tilde{\boldsymbol{v}}^{(i)}}{\partial t}+\left(\tilde{\boldsymbol{v}}^{(i)} \cdot \boldsymbol{\nabla}\right) \tilde{\boldsymbol{v}}^{(i)}\right)=\boldsymbol{\nabla} \cdot \tilde{\boldsymbol{T}}^{(i)}+\rho_{i} \boldsymbol{F},
$$

where $\boldsymbol{F}$ is the vector of the external body forces, $\rho_{i}$ is the density and $\tilde{\boldsymbol{T}}^{(i)}$ is the stress tensor of the $i$ th liquid.

The kinematic and dynamic boundary conditions at the part $\tilde{\Gamma}_{i j}^{r}$ of the free interface $\Gamma_{i j}$ lying in the outer region (see figure 2 ) are respectively

and

$$
\left.\left(\tilde{\boldsymbol{v}}^{(i)}-\tilde{\boldsymbol{v}}^{(j)}\right)\right|_{\tilde{\Gamma}_{i j}^{r}}=0
$$

$$
\left(\tilde{\boldsymbol{T}}^{(i)}-\tilde{\boldsymbol{T}}^{(j)}\right) \cdot \boldsymbol{n}=\sigma_{i j}\left(\frac{1}{R_{1}}+\frac{1}{R_{2}}\right) \boldsymbol{n},
$$

where $\boldsymbol{n}$ is the unit normal vector to $\tilde{\Gamma}_{i j}^{r}, \sigma_{i j}$ is the surface tension coefficient, $R_{1}$ and $R_{2}$ are the principle radii of curvature.

The no-slip boundary condition at $\tilde{\Gamma}_{i 3}^{r}$ is applied in the L-F-S case:

$$
\left.\tilde{\boldsymbol{v}}\right|_{\tilde{\Gamma}_{i 3}^{r}}=\boldsymbol{v}_{s},
$$

where $\boldsymbol{v}_{s}$ is the velocity of the solid phase.

If some of the fluid is unbounded or an unsteady problem is considered then the corresponding boundary and initial conditions have to be applied. These conditions are specified in $\S \S 6$ and 7, where concrete problems are considered. To close the above mathematical model kinematic and dynamic conditions as well as the slope of the free interfaces at the boundary $\left(\partial \Omega^{r}\right)$ between the inner and the outer regions are required. To formulate these conditions the inner region is considered in the next section.

\section{The inner region}

The main goal in this section is to obtain appropriate integral characteristics of the dynamics in the inner region and, using them, to take into account their influence on the outer region. These integral characteristics are obtained on the basis of mass and momentum conservation in the inner region. Let us consider first the more general case: three immiscible liquids are in mutual contact and $\Omega^{r}$ is a region where any two 
$(a)$

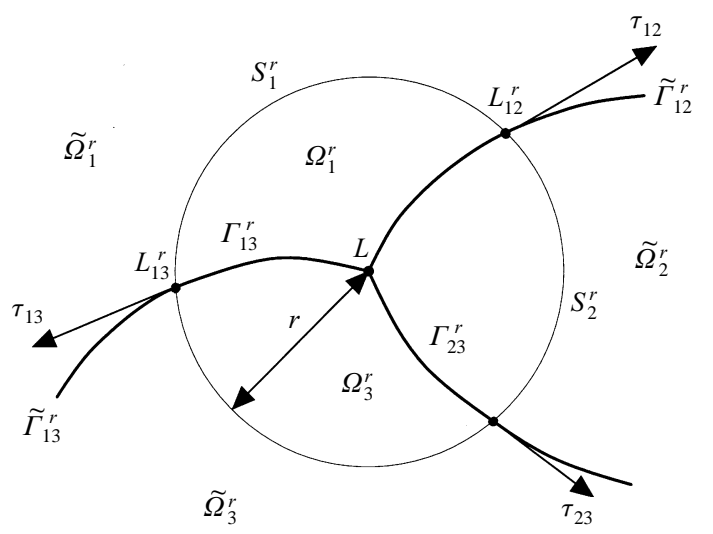

(b)

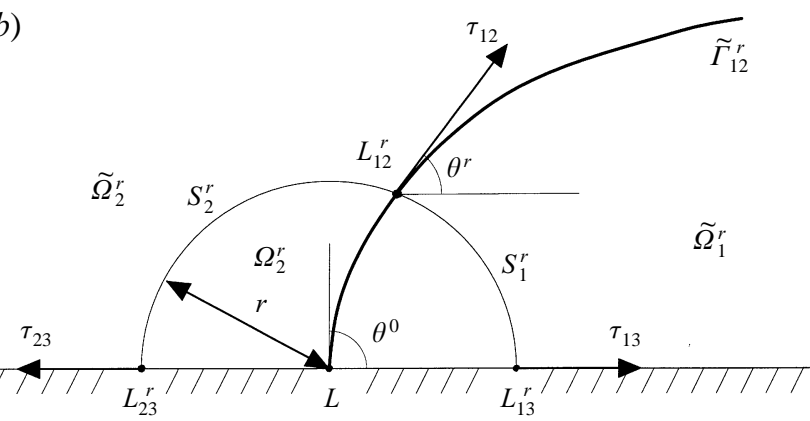

FIGURE 2. Schematic diagram of the inner and outer regions: (a) a liquid-fluid-liquid contact line, (b) a liquid-fluid-solid contact line.

of them influence each other ( $\Omega^{r}$ is called the contact or inner region). To the end of the paper $\Omega^{r}$ will be considered as a deformable region, the bounding surface of which follows the flow, so there is no flow through $\partial \Omega^{r}$. For this choice of $\Omega^{r}$ the conservation equations can be written in a more compact form and also it corresponds to the Lagrangian approach used at the numerical simulation (see $\S 6$ ).

The mass conservation equation of the $i$ th liquid in the inner region is

$$
\frac{\mathrm{d} m_{i}^{r}}{\mathrm{~d} t}=0
$$

where $m_{i}^{r}$ is the mass of $i$ th liquid in $\Omega^{r}$.

Momentum conservation in the inner region is, in its most fundamental form a relation equating the rate of change of momentum of the fluids in $\Omega^{r}$ and the sum of all forces acting on the fluids in this region:

$$
\frac{\mathrm{d} \boldsymbol{M}^{r}}{\mathrm{~d} t}-\boldsymbol{F}^{r}=\sum_{i=1}^{3} \int_{S_{i}^{r}} \tilde{\boldsymbol{T}}^{(i)} \cdot \boldsymbol{n} \mathrm{d} s+\left.\sum_{\substack{i, j=1 \\ i<j}}^{3}\left(\sigma_{i j} \tau_{i j}\right)\right|_{L_{i j}^{r},},
$$

where $\boldsymbol{M}^{r}$ and $\boldsymbol{F}^{r}$ are respectively the vectors of momentum and body forces in $\Omega^{r}$. The first sum represents the surface forces exerted on the inner region across its boundary

$$
\partial \Omega^{r}=\bigcup_{i=1}^{3} S_{i}^{r}
$$

where $\tilde{\boldsymbol{T}}^{(i)}$ are the stress tensors in the outer region. The last sum in (4.2) represents so- 
called shell or capillary forces (see Nigmatulin 1978; Sullivan \& Telo da Gama 1986 and references therein), where $\tau_{i j}$ is the unit tangent to the interface $\widetilde{\Gamma}_{i j}$ directed outwards from $\Omega^{r}$ (see figure $2 a$ ) and $\sigma_{i j}$ is the surface tension coefficient of the interface $\Gamma_{i j}$.

In the $\mathrm{L}-\mathrm{F}-\mathrm{S}$ case $\left(\Omega_{3}\right.$ is the solid phase) the solid surface is assumed to be smooth and locally plane $\left(\tau_{13}^{r}=-\tau_{23}^{r}\right)$. Then the normal (with respect to the solid surface) component of (4.2) is balanced by the reaction force of the wall and consequently momentum conservation in this case can be written as

$$
\boldsymbol{\tau} \cdot\left(\frac{\mathrm{d} \boldsymbol{M}^{r}}{\mathrm{~d} t}-\boldsymbol{F}^{r}\right)-\boldsymbol{\tau} \cdot \sum_{i=1}^{2} \boldsymbol{W}_{i 3}^{r}=\boldsymbol{\tau} \cdot \sum_{i=1}^{2} \int_{S_{i}^{r}} \tilde{\boldsymbol{T}}^{(i)} \cdot \boldsymbol{n} \mathrm{d} s+\boldsymbol{\tau} \cdot \sum_{\substack{i, j=1 \\ i<j}}^{3}\left(\sigma_{i j} \tau_{i j}\right)_{\mid L_{i j}^{r}},
$$

where $\tau$ is the tangential vector to the solid surface, and $W_{i 3}^{r}$ is the force which the solid exerts on the $i$ th liquid across $\Gamma_{i 3}^{r}$ (see figure $2 b$ ).

Remark. In writing equations (4.2) and (4.3) no restrictions on the fluids in the inner region are imposed. The values of $\sigma_{i j}, \tau_{i j}$ and $\tilde{\boldsymbol{T}}^{(i)}$ which appear in these equations are assumed to be well defined in the outer region.

The purpose is to obtain a solution in the outer region, which satisfies the conservation equations (4.1) and (4.2) or (4.3). There would not be problems if the lefthand sides of (4.2) and (4.3) were known, which unfortunately is not the case because of the difficulties arising from modelling of the contact-line region. An alternative approach is to approximate the unknown terms in appropriate way. There are several possibilities for evaluating the left-hand sides of these equations. The simplest one is to assume that they are zero; however, this is equivalent to the assumption that the inner and outer regions do not influence each other. Another possibility is to assume that some model is valid in most of the inner region and then by matching the solutions for both regions an appropriate solution in the outer region could be obtained. Following this idea the main assumption in the present paper is that Navier-Stokes equations describe the fluid flow in most of the inner region $\left(\Omega^{r} \backslash \Omega^{\varepsilon} ; \varepsilon \ll r\right)$ for each of the fluids. In this case the left-hand sides of (4.1)-(4.3) can be evaluated as follows:

$$
\begin{aligned}
& \frac{\mathrm{d} m_{i}^{r}}{\mathrm{~d} t}=\rho_{i} \int_{\Omega_{i}^{r}} \boldsymbol{\nabla} \cdot \boldsymbol{v}^{(i)} \mathrm{d} \Omega+O\left((\varepsilon / r)^{2}\right), \\
& \frac{\mathrm{d} \boldsymbol{M}^{r}}{\mathrm{~d} t}-\boldsymbol{F}^{r}+O\left((\varepsilon / r)^{2}\right)=\sum_{i=1}^{k} \rho_{i} \int_{\Omega_{i}^{r}}\left(\frac{\mathrm{D} \boldsymbol{v}^{(i)}}{\mathrm{D} t}-\boldsymbol{F}\right) \mathrm{d} \Omega \\
&=\sum_{i=1}^{k} \int_{\Omega_{i}^{r}} \boldsymbol{\nabla} \cdot \boldsymbol{T}^{(i)} \mathrm{d} \Omega=\sum_{i=1}^{k} \int_{\partial \Omega_{i}^{r}} \boldsymbol{T}^{(i)} \cdot \boldsymbol{n} \mathrm{d} s
\end{aligned}
$$

where $k$ is the number of fluids involved.

The force balance condition at the boundary between the inner and the outer regions is

$$
\int_{S_{i}^{r}}\left(\boldsymbol{T}^{(i)}-\tilde{\boldsymbol{T}}^{(i)}\right) \cdot \boldsymbol{n}_{i} \mathrm{~d} s=O(\varepsilon / r)
$$

where $\boldsymbol{n}_{i}$ is normal vector to $S_{i}^{r}$, and $\boldsymbol{T}^{(i)}$ and $\tilde{\boldsymbol{T}}^{(i)}$ are the stress tensors in the inner and the outer regions respectively.

In the case of the $\mathrm{L}-\mathrm{F}-\mathrm{S}$ contact line condition (4.6) at the solid surface takes the form

$$
W_{i 3}^{r}=\int_{\Gamma_{i 3}^{r}} \boldsymbol{T}^{(i)} \cdot \boldsymbol{n}_{i} \mathrm{~d} s+O(\varepsilon / r)
$$


Remark. The errors $O\left((\varepsilon / r)^{2}\right)$ and $O(\varepsilon / r)$ in the above evaluations appear because of the region $\Omega^{\varepsilon}$, where Navier-Stokes equations are not applicable. The evaluations (4.4)-(4.7) are not deduced strictly, they are only assumptions and will be checked by means of numerical tests and comparisons with experimental results.

It is easy to see that if the left-hand sides of (4.1) and (4.2) are evaluated by means of (4.4) and (4.5) and using (4.6), then (4.1) and (4.2) can be approximated with error $O(\varepsilon / r)$ by means of integral relations:

mass conservation

$$
\int_{\Omega_{i}^{r}} \boldsymbol{\nabla} \cdot \boldsymbol{v}^{(i)} \mathrm{d} \Omega=0
$$

momentum conservation for the $\mathrm{L}-\mathrm{F}-\mathrm{L}$ contact line

$$
\sum_{\substack{i, j=1 \\ i<j}}^{3}\left(\int_{\Gamma_{i j}^{r}}\left(\boldsymbol{T}^{(i)}-\boldsymbol{T}^{(j)}\right) \cdot \boldsymbol{n}_{i j} \mathrm{~d} s-\left.\left(\sigma_{i j} \cdot \tau_{i j}\right)\right|_{L_{i j}^{r}}\right)=0,
$$

where $\boldsymbol{n}_{i j}$ is the unit normal to $\Gamma_{i j}^{r}$ vector outward from $\Omega_{j}^{r}$,

$$
\partial \Omega_{i}^{r}=\Gamma_{i j}^{r} \cup \Gamma_{i l}^{r} \cup S_{i}^{r}(i \neq j \neq l \neq i) ;
$$

momentum conservation for the $\mathrm{L}-\mathrm{F}-\mathrm{S}$ contact line, where the difference $\sigma_{13}-\sigma_{23}$ is expressed by means of the static boundary condition (1.2) and then (4.3) becomes

$$
\boldsymbol{\tau} \cdot \int_{\Gamma_{12}^{r}}\left(\boldsymbol{T}^{(1)}-\boldsymbol{T}^{(2)}\right) \cdot \boldsymbol{n}_{12} \mathrm{~d} s-\sigma_{12}\left(\cos \left(\theta_{s}\right)-\cos \left(\theta^{r}\right)\right)=0,
$$

where $\tau=\tau_{13}=-\tau_{23}, \theta_{s}$ is the static contact angle (see figure $1 b$ ) and $\theta^{r}$ is the inclination at a distance $r$ from the contact line $\left(\cos \left(\theta^{r}\right)=\tau \cdot \tau_{12}^{r}\right.$, see figure $\left.2 b\right)$.

Equations (4.6), (4.9) and (4.10) can be used to match the solution of the differential equations in the outer region to a solution of the integral equations in the inner one ((4.8) and the right-hand sides of (4.5)). The formulation of the problem in the entire flow region is described in $\S 5$, where the correctness of this approach is discussed.

\section{Mathematical formulation of the problem}

In this section the equations of motion and the boundary conditions in the outer $\left(\tilde{\Omega}^{r}\right)$ and the inner $\left(\Omega^{r}\right)$ regions as well as the matching condition at the boundary between them are formulated. Two immiscible liquids and a fluid in the $\mathrm{L}-\mathrm{F}-\mathrm{L}$ case or a liquid, a fluid and a solid in the $\mathrm{L}-\mathrm{F}-\mathrm{S}$ one, which are in mutual contact are considered.

It is assumed that the liquids are homogeneous, incompressible, Newtonian, with constant viscosities $\mu_{i}$ and densities $\rho_{i}$, and the solid is rigid and its surface is smooth. The surface tension coefficient $\sigma_{i j}$ in the outer region is assumed to be constant. There are no assumptions about $\left\{\sigma_{i j}\right\}$ in the inner region. The gravity force is considered as a example of an external body force.

To write the equations and the boundary conditions in dimensionless form the characteristic length $l$ and velocity $U_{0}$ are chosen. Their values are specified in $\S \S 6$ and 7 where the numerical results are presented. Reynolds, Weber and Froude numbers are denoted by

$$
R e=\frac{\rho_{1} U_{0} l}{\mu_{1}} ; \quad W e=\frac{\rho_{1} U_{0}^{2} l}{\sigma_{12}}, \quad F r=\frac{U_{0}^{2}}{g l}
$$


The density, viscosity and surface tension coefficient ratios are

$$
\lambda_{i}=\rho_{i} / \rho_{1} ; \quad \eta_{i}=\mu_{i} / \mu_{1} \text { and } \kappa_{i j}=\sigma_{i j} / \sigma_{12} .
$$

The motion of the $i$ th liquid in the outer region, $\tilde{\Omega}^{r}$, is described by the dimensionless Navier-Stokes equations

$$
\lambda_{i}\left(\frac{\mathrm{D} \tilde{\boldsymbol{v}}^{(i)}}{\mathrm{D} t}-\boldsymbol{F}\right)=\nabla \cdot \tilde{\boldsymbol{T}}^{(i)}
$$

and the continuity equations

$$
\begin{gathered}
\boldsymbol{\nabla} \cdot \tilde{\boldsymbol{v}}^{(i)}=0, \\
\tilde{\boldsymbol{T}}^{(i)}=-\tilde{p}^{(i)} \boldsymbol{I}+\frac{\eta_{i}}{\operatorname{Re}} \tilde{\boldsymbol{E}}^{(i)}, \\
\tilde{\boldsymbol{E}}_{k l}^{(i)} \boldsymbol{v}=0.5\left(\frac{\partial \tilde{\boldsymbol{v}}_{k}^{(i)}}{\partial x_{l}}+\frac{\partial \tilde{\boldsymbol{v}}_{l}^{(i)}}{\partial x_{k}}\right)
\end{gathered}
$$

where $\boldsymbol{I}$ is the unit tensor, $p$ is pressure, $\boldsymbol{F}=(0 ;-1 / F r)$ and $\lambda_{1}=\eta_{1}=1$.

The boundary conditions at that part $\tilde{\Gamma}_{i j}^{r}$ of the liquid-liquid interface $\Gamma_{i j}$ lying in the outer region are (see (3.3) and (3.4))

$$
\left(\tilde{\boldsymbol{T}}^{(i)}-\tilde{\boldsymbol{T}}^{(j)}\right) \cdot \boldsymbol{n}=\left(\kappa_{i j} / W e\right)\left(1 / R_{1}+1 / R_{2}\right) \boldsymbol{n}
$$

If the $i$ th phase is gas then

$$
\tilde{\boldsymbol{T}}^{(i)}=-p_{0}(t) \boldsymbol{l},
$$

i.e. it is assumed that the gas is ideal, incompressible and the pressure in it is constant with respect to the space variables:

$$
\left.\left(\tilde{\boldsymbol{v}}^{(i)}-\tilde{\boldsymbol{v}}^{(j)}\right)\right|_{\tilde{\Gamma}_{i j}^{r}}=0 .
$$

In the inner region the integral Navier-Stokes equations

$$
\int_{\Omega_{i}^{r}}\left(\lambda_{i}\left(\frac{\mathrm{D} \boldsymbol{v}^{(i)}}{\mathrm{D} t}-\boldsymbol{F}\right)-\boldsymbol{\nabla} \cdot \boldsymbol{T}^{(i)}\right) \mathrm{d} \Omega=0
$$

and the continuity equation

are solved.

$$
\int_{\Omega_{i}^{r}} \boldsymbol{\nabla} \cdot \boldsymbol{u}^{(i)} \mathrm{d} \Omega=0
$$

The matching conditions at the boundary $\partial \Omega^{r}=S_{1}^{r} \cup S_{2}^{r} \cup S_{3}^{r}$ between the regions are

dynamic conditions

$$
\left.\left(\boldsymbol{T}^{(i)} \cdot \boldsymbol{n}_{i}-\tilde{\boldsymbol{T}}^{(i)} \cdot \boldsymbol{n}_{i}\right)\right|_{S_{i}^{r}}=0,
$$

where $\boldsymbol{n}_{i}$ is a normal vector to $S_{i}^{r}$;

kinematic conditions

The equations

$$
\left.\left(\boldsymbol{v}^{(i)}-\tilde{\boldsymbol{v}}^{(i)}\right)\right|_{S_{i}^{r}}=0
$$

$$
\sum_{\substack{i, j=1 \\ i<j}}^{3} \int_{\Gamma_{i j}^{r}}\left(\boldsymbol{T}^{(i)}-\boldsymbol{T}^{(j)}\right) \cdot \boldsymbol{n}_{i j} \mathrm{~d} s-\left.\frac{1}{W e} \sum_{\substack{i, j=1 \\ i<j}}^{3}\left(\kappa_{i j} \tau_{i j}\right)\right|_{L_{i j}^{r}}=0
$$


in the case of the $\mathrm{L}-\mathrm{F}-\mathrm{L}$ contact line (see also (4.9)) and

$$
\tau \cdot \int_{\Gamma_{12}^{r}}\left(\boldsymbol{T}^{(1)}-\boldsymbol{T}^{(2)}\right) \cdot \boldsymbol{n}_{12} \mathrm{~d} s-\frac{1}{W e}\left(\cos \left(\theta_{s}\right)-\cos \left(\theta^{r}\right)\right)=0
$$

in the $\mathrm{L}-\mathrm{F}-\mathrm{S}$ one (see also (4.10)) can be considered as integral boundary conditions at $\Gamma_{i j}^{r}$ for the equations in the inner region (if $\tau_{i j}$ or $\theta^{r}$ are known) or as a geometrical ones at the points $L_{i j}$ for the equations in the outer region (if $\boldsymbol{T}^{(k)}$ and $\Gamma_{i j}^{r}$ are known). Actually (5.10)-(5.13) are the matching condition between the inner $\left(\boldsymbol{v}, p, \Gamma_{i j}^{r}\right)$ and outer $\left(\tilde{\boldsymbol{v}}, \tilde{p}, \tilde{\Gamma}_{i j}^{r}\right)$ solutions.

In the case of $\mathrm{L}-\mathrm{F}-\mathrm{S}$ contact-line problems the boundary conditions at the solid surface are

no-slip far from the contact line

$$
\left.\boldsymbol{v}^{(i)}\right|_{\tilde{\Gamma}_{i 3}^{\beta}}=\boldsymbol{v}_{s},
$$

where $\beta$ is the slip length and $\boldsymbol{v}_{s}$ is the velocity of the solid surface;

slip on that part $\Gamma_{i 3}^{\beta}$ of the solid surface in the contact-line vicinity with radius $\beta$ :

$$
\left.\boldsymbol{v}^{(i)}\right|_{L_{i 3}^{\alpha}}=\left((\beta-\alpha) \boldsymbol{v}_{L}+\alpha \boldsymbol{v}_{s}\right) / \beta, \quad \alpha \in[0, \beta],
$$

i.e. linear variation of $\boldsymbol{v}^{(i)}$ on the segment $L_{i 3}^{\beta} L$, where $\boldsymbol{v}_{L}$ is the contact-line velocity.

It is evident that in this $\mathrm{L}-\mathrm{F}-\mathrm{S}$ case the inner solution, in particular $\theta^{r}$, will depend on the slip length $\beta$ and consequently the outer solution will also depend on the slip condition applied.

When an unsteady problem is considered the free interface $\Gamma_{i j}$ satisfies the kinematic condition

$$
\frac{\mathrm{D} S_{i j}}{\mathrm{D} t}=\frac{\partial S_{i j}}{\partial t}+\boldsymbol{v} \cdot \nabla S_{i j}=0
$$

where $S_{i j}(x, t)=0$ is the equation of the free interface $\Gamma_{i j}$. Conditions for quiescent liquids and spherical free interfaces

$$
\boldsymbol{v}(x, 0)=0, \quad S_{i j}(x, 0)=S_{i j}^{0}(x),
$$

where $S_{i j}^{0}(x)=0$ is the equation of a sphere, are used as initial conditions in the majority of the cases considered in this paper. Of course, initial conditions different from (5.17) can be used if there exists information about $v(x, 0)$ and the position of $\Gamma_{i j}$ at $t=0$.

The key to the correctness of this approach (the uniqueness of the solution $(\tilde{\boldsymbol{v}}, \tilde{p}, \tilde{\Gamma})$ in $\tilde{\Omega}^{r}$ ) lies in the assumption that different solutions of the integral equations (5.8) and (5.9) (the inner solutions) 'prematch' the solution in the outer region. This assumption is numerically verified in the next section for a problem containing a $\mathrm{L}-\mathrm{F}-\mathrm{L}$ contact line.

It is easy to see that, under the above assumption, if $(\tilde{\boldsymbol{v}}, \tilde{p}, \tilde{\Gamma})^{r_{1}}$ and $(\tilde{\boldsymbol{v}}, \tilde{p}, \tilde{\Gamma})^{r_{2}}$ are two solutions for two outer regions $\tilde{\Omega}^{r_{1}}$ and $\widetilde{\Omega}^{r_{2}}$ respectively, then these solutions are equivalent in the common part of both regions, i.e. $(\tilde{\boldsymbol{v}}, \tilde{p}, \tilde{\Gamma})^{r_{1}}=(\tilde{\boldsymbol{v}}, \tilde{p}, \tilde{\Gamma})^{r_{2}}$ in $\widetilde{\Omega}^{r_{1}} \cap \widetilde{\Omega}^{r_{2}}$. In other words the choice of $r$ does not influence the outer solution.

To determine the appropriate equivalent radius $r$ of the inner region one can proceed from (i) the above conclusion that the outer solution of the problem (5.1)-(5.17) does 
not depend on the value of $r$; (ii) the solution of the integral Navier-Stokes and continuity equations (5.8) and (5.9) approximate well the actual momentum and mass conservation in the inner region, i.e. $\varepsilon / r$ is small. $\varepsilon$ is the equivalent radius of the region around the contact line where the outer mathematical model (5.1)-(5.7) is not valid (see $\S 4)$. Thus $r$ should be assigned a value larger than the range of intermolecular forces $(\varepsilon)$, but much smaller than the characteristic length scale $l$ of the problem (see also Boender et al. 1991).

It is very difficult, however, to evaluate the error which arises on replacing the actual inertial and body forces with these obtained using (5.8). The distance from the contact line up to which this approach gives good results can be evaluated by comparing the numerical results with experimental ones very close to the contact line.

\section{Numerical method}

In the present section a brief description of the numerical method is given. It is a divergence-free finite-element method in velocity-pressure variables. The finite elements are isoparametric quadrilaterals with a 9-node quadratic approximation for the velocity and linear one for the pressure. In the case of unsteady problems the time integration is performed by means of a Lagrangian approach. The method is described in detail in Shopov, Minev \& Bazhlekov (1992) for unsteady hydrodynamic problems with free surfaces and its development for problems containing dynamic contact lines can be found in Bazhlekov \& Shopov (1990) and Shopov \& Bazhlekov (1991).

In terms of the numerical method used here there is no difference between the inner and outer regions - equations (5.1)-(5.17) are discretized and solved in the whole liquid regions. However, while the solution near to the contact line (inner region) depends on the finite-element mesh used, at a distance from it (outer region) the solution does not depend on the mesh. This is shown at the end of this section for a concrete $\mathrm{L}-\mathrm{F}-\mathrm{L}$ contact-line problem.

The velocity, pressure and free-surface positions are determined by requiring that the weighted residuals vanish:

$$
\begin{gathered}
\sum_{i=1}^{k} \int_{\Omega_{i}}\left(\lambda_{i}\left(\frac{\mathrm{D} \boldsymbol{v}^{(i)}}{\mathrm{d} t}-\boldsymbol{F}\right) \cdot \gamma \boldsymbol{v}+\boldsymbol{T}^{(i)} \cdot \boldsymbol{E}^{(i)} \gamma \boldsymbol{v}\right) \mathrm{d} \Omega=\sum_{i=1}^{k} \int_{\partial \Omega_{i}} \boldsymbol{n} \cdot \boldsymbol{T}^{(i)} \cdot \gamma \boldsymbol{v} \mathrm{d} s \\
\int_{\Omega_{i}}\left(\boldsymbol{\nabla} \cdot \boldsymbol{v}^{(i)}\right) \gamma p \mathrm{~d} \Omega=0, \quad i=1, \ldots, k,
\end{gathered}
$$

where $k$ is the number of liquids involved, $\boldsymbol{n}$ is the unit normal to $\partial \Omega_{i}, \gamma \boldsymbol{v}$ and $\gamma p$ are weighted functions for velocity and pressure respectively. Equation (6.1) is obtained by applying the divergence theorem to the terms $\boldsymbol{\nabla} \cdot \boldsymbol{T}^{(i)}$ of (5.1) for the $i$ th liquid after it has been weighted by $\gamma \boldsymbol{v}$ and integrated over the whole domain $\Omega_{i}$ and summing by the index $i$ of the liquid.

The essential boundary conditions (5.14) and (5.15) are satisfied by the choice of the basis functions (see Shopov et al. 1992). The natural boundary conditions (5.5) are imposed by replacing the terms $\left(\boldsymbol{T}^{(i)}-\boldsymbol{T}^{(j)}\right) \cdot \boldsymbol{n}$ at the inner points of $\Gamma_{i j}$ on the righthand side of (6.1) with $\left(\kappa_{i j} / W e\right)\left(1 / R_{1}+1 / R_{2}\right) n$. At the contact line where (5.5) is not satisfied (the main curvatures are not defined) there are several possibilities.

(i) To assume that the contribution of $\left.\left(\boldsymbol{T}^{(i)} \cdot \boldsymbol{n}\right)\right|_{L}$ on the right-hand side of (6.1) is zero. Such an approach, used by Silliman \& Scriven (1980), occurs if the terms of the tensors $\boldsymbol{T}^{(i)}$ are finite and corresponds to the static boundary conditions (1.1) and (1.2), as shown below. 
(ii) In this paper the possibility that the contribution of $\left(\boldsymbol{T}^{(i)} \cdot \boldsymbol{n}\right)_{\mid L}$ in (6.1) can be nonzero is considered and consequently it must be taken into account. To evaluate this contribution it is assumed that: $(a) \Gamma_{i j}$ are smooth up to the contact line, i.e. the limits

exists; $(b)$

$$
\lim _{r \rightarrow \mathbf{0}}\left(\tau_{i j}\left(L_{i j}^{r}\right)\right)=\tau_{i j}(L) \quad \text { and } \quad \lim _{r \rightarrow \mathbf{0}}\left(n_{i j}\left(L_{i j}^{r}\right)\right)=n_{i j}(L)
$$

$$
\lim _{r \rightarrow 0} \int_{\Gamma_{i j}^{r}} \boldsymbol{T}^{(i)} \cdot \boldsymbol{n}_{i j} \mathrm{~d} s
$$

exists and it can be non-zero. This is the case if the tensor $\boldsymbol{T}^{(i)}{ }_{L L}$ contains infinite terms, but $\boldsymbol{T}^{(i)} \cdot \boldsymbol{n}$ is integrable, i.e. the stress at the contact line can be infinite but the forces are finite, $\dagger$ which is physically reasonable as has been pointed out by Dussan V. \& Davis (1974).

Under these assumptions where $r$ tends to zero equations (5.12) and (5.13) become respectively

$$
\begin{gathered}
\left.\left(\sum_{\substack{i, j=1 \\
i<j}}^{3}\left(\boldsymbol{T}^{(i)}-\boldsymbol{T}^{(j)}\right) \cdot \boldsymbol{n}_{i j}\right)\right|_{L}=\left.\frac{2 \delta_{L}}{W e}\left(\sum_{\substack{i, j=1 \\
i<j}}^{3}\left(\kappa_{i j} \tau_{i j}\right)\right)\right|_{L}, \\
\left.\boldsymbol{\tau} \cdot\left(\boldsymbol{T}^{(1)}-\boldsymbol{T}^{(2)}\right) \cdot \boldsymbol{n}_{12}\right|_{L}=\frac{2 \delta_{L}}{W e}\left(\cos \left(\theta_{s}\right)-\cos \left(\theta^{\circ}\right)\right),
\end{gathered}
$$

where $\delta_{L}$ is Dirac's function and $\theta^{\circ}=\left.\theta^{r}\right|_{r=0}\left(\cos \left(\theta^{\circ}\right)=\tau(L) \cdot \tau_{12}(L)\right)$. Equations (6.3) and (6.4) differ from the static boundary conditions (1.1) and (1.2) if any of the terms $\boldsymbol{T}^{(i)} \cdot \boldsymbol{n}$ are infinite, but they are reduced to the static equations (1.1) and (1.2) in the static case, since in this case the $\boldsymbol{T}^{(i)} \cdot \boldsymbol{n}$ are finite.

Remark. Equations (6.3) and (6.4) have no direct physical meaning, and because of that they are not included in the mathematical model (5.1)-(5.17) and their deduction is not discussed in detail.

Replacing the terms $\boldsymbol{T}^{(i)} \cdot \boldsymbol{n}$ on the right-hand side of (6.1) by equations (5.5), (6.3) and (6.4) we obtain

$$
\sum_{i=1}^{k} \int_{\Omega_{i}}\left(\lambda_{i}\left(\frac{\mathrm{D} \boldsymbol{v}^{(i)}}{\mathrm{D} t}-\boldsymbol{F}\right) \cdot \gamma \boldsymbol{v}+\boldsymbol{T}^{(i)} \cdot \boldsymbol{E}^{(i)} \gamma \boldsymbol{v}\right) \mathrm{d} \Omega=\sum_{i, j} \int_{\Gamma_{i j}} \boldsymbol{R}_{i j} \cdot \gamma \boldsymbol{v} \mathrm{d} s+\phi_{L}(\gamma \boldsymbol{v})
$$

where

$$
\boldsymbol{R}_{i j}=\frac{\kappa_{i j}}{W e}\left(\frac{1}{R_{1}}+\frac{1}{R_{2}}\right) \boldsymbol{n}
$$

and

$$
\phi_{L}(\gamma \boldsymbol{v})= \begin{cases}\left.\frac{1}{W e}\left(\kappa_{12} \tau_{12}+\kappa_{13} \tau_{13}+\kappa_{23} \tau_{23}\right)\right|_{L} \cdot \gamma \boldsymbol{v}(L) & \mathrm{L}-\mathrm{F}-\mathrm{L} \text { case } \\ \frac{1}{W e}\left(\cos \left(\theta_{s}\right)-\cos \left(\theta^{\circ}\right)\right) \cdot \gamma \boldsymbol{v}(L) \cdot \tau(L) & \mathrm{L}-\mathrm{F}-\mathrm{S} \text { case }\end{cases}
$$

It is easy to see that approach (ii) is reduced to (i) when $\phi_{L}=0$ (actually $\phi_{L}=0$ is the static boundary condition). This gives us grounds for concluding that the approach described in this paper is more general than that used by Silliman \& Scriven (1980). On including the term $\phi_{L}$ in (6.5) the integral equations (5.13) and (5.14), which

$\dagger$ In Shopov \& Bazhlekov (1991) there is an error: the words 'infinite forces' should read 'infinite stress'. 


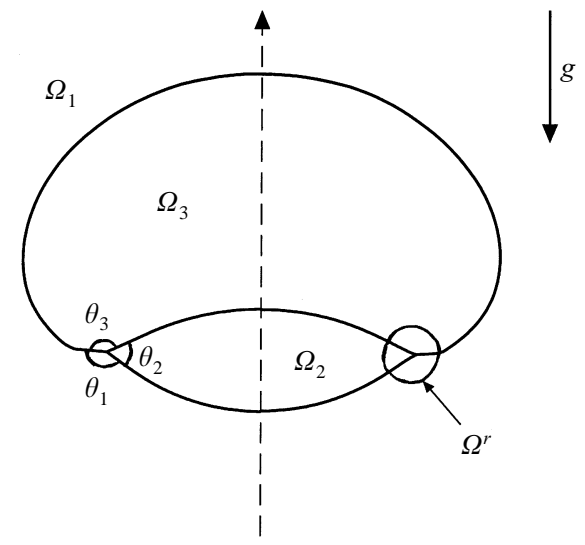

FIGURE 3. Comparison between two steady shapes of a $3 \mathrm{~S}$ compound drop obtained using meshes A (left) and C (right).

approximate momentum conservation in the inner region, are satisfied. It is easy to see that, when $\phi_{L}$ is not included in (6.5), these equations are satisfied only if the static boundary conditions (1.1) and (1.2) are imposed at the contact line. Thus Christodoulou \& Scriven (1989), investigating slide coating numerically, have obtained dynamic contact angles different from those that had been imposed. The reason is the terms similar to $\phi_{L}$ in their equation (38). Geometrical condition such as (1.1) and (1.2) are extra for the problem (6.5) and they cannot always be satisfied. Furthermore, the contact angle obtained sometimes depends substantially on the mesh and especially on the spatial step in the contact line vicinity, which is demonstrated numerically in the example considered below.

The main assumption of the approach described, that the different solutions of the integral equations (5.8) and (5.9) in the inner region 'prematch' one and the same solution in the outer one is tested numerically by considering the problem of steady free rise of a $3 \mathrm{~S}$ gas-liquid compound drop in a gravitational field. This $3 \mathrm{~S}$ compound drop represents a gas bubble $\left(\Omega_{3}\right)$ partially covered by a liquid drop $\left(\Omega_{2}\right)$ (see figure 3 ). In this case a dynamic liquid-gas-liquid contact line is formed. The compound drop equivalent radius, $l$, is chosen to be the characteristic length. Stokes terminal velocity $U_{0}=\frac{2}{9}\left(l^{2} g\left|\rho_{1}-\rho_{a}\right| / \mu_{1}\right)$, for a solid sphere with a density equal to the average compound drop density $\rho_{a}=\rho_{3}(1-e)+\rho_{2} e$ and radius $l$, is chosen to be the characteristic velocity. The independent dimensionless parameters are $R e, W e, \eta_{2}=$ $\mu_{2} / \mu_{1}, \lambda_{2}=\rho_{2} / \rho_{1}, \kappa_{13}=\sigma_{13} / \sigma_{12}, \kappa_{23}=\sigma_{23} / \sigma_{12}$, and $e=\operatorname{vol}\left(\Omega_{2}\right) /\left(\operatorname{vol}\left(\Omega_{3} \cup \Omega_{2}\right)-\right.$ the ratio between the volumes of the liquid phase of the compound drop and the whole drop. $R e$ is Reynolds number, We is Weber number and the Froude number is expressed as $F r=(2 / 9) \operatorname{Re}\left(1-\lambda_{3}(1-e)-\lambda_{2} e\right)$. In this case, $\Omega_{3}$ is gas, so $\rho_{3}=\lambda_{3}=0$.

In the case considered below the dimensionless radius of the inner region is $r=0.12$. Three different finite-elements meshes, A, B, and C, are considered. The inner region of the first discretization, A, consists of one layer of finite elements around the contact line (i.e. the spatial step $h_{\mathrm{A}}$ in the inner region is about 0.12 ). The mesh $\mathrm{B}$ is obtained from $\mathrm{A}$ by dividing each element in the inner region into four new elements, and $\mathrm{C}$ is obtained from B in the same manner. So the spatial steps in the inner region are as follows: $h_{\mathrm{A}}=0.12, h_{\mathrm{B}}=0.06$ and $h_{\mathrm{C}}=0.03$. Using these three meshes the unsteady problem is solved until the compound drop steady shape is obtained.

The test is conducted at $R e=5, W e=10, \eta_{2}=2.5, \lambda_{2}=0.5, \kappa_{13}=2, \kappa_{23}=1$, and $e=0.11$. At these values of $\kappa_{13}=2$ and $\kappa_{23}=1$ it is evident that $\sigma_{13}=2 \sigma_{12}$ and 


$\begin{array}{cccccc}\text { Mesh } & \begin{array}{c}\text { Spatial } \\ \text { step } h\end{array} & \begin{array}{c}\theta_{1} \\ \text { (deg.) }\end{array} & \begin{array}{c}\theta_{2} \\ \text { (deg.) }\end{array} & \begin{array}{c}\theta_{3} \\ (\text { deg. })\end{array} & R_{L} \\ \text { A } & 0.12 & 154 & 57 & 149 & 0.740 \\ \text { B } & 0.06 & 158 & 47 & 155 & 0.746 \\ \text { C } & 0.03 & 161 & 40 & 159 & 0.749\end{array}$

TABLE 1. Numerical results for the contact angles obtained at the contact line and the position $R_{L}$ of the contact line.

$\sigma_{23}=\sigma_{12}$ and consequently $\sigma_{13}=\sigma_{12}+\sigma_{23}$, i.e. the static contact angles are $\theta_{1}=180^{\circ}$, $\theta_{2}=0^{\circ}, \theta_{3}=180^{\circ}$ and consequently no static shape of the compound drop exists in the configuration including contact line.

In table 1 the numerical results for the contact angles obtained at the contact line as well as the position $R_{L}$ of the contact line are shown $\left(R_{L}\right.$ is the distance between the contact line and the axes of symmetry). It is evident that the contact angles obtained, and consequently the free interface shapes near the contact line, depend on the spatial step used in the inner region. At the same time, however, the contact-line position, $R_{L}$, and the compound drop shape in the outer region are insensitive to the mesh. This can be seen on figure 3, where two shapes of the compound drop are shown, the left-hand one obtained using mesh $\mathrm{A}$ and the right-hand one using $\mathrm{C}$.

The above-described test confirms the main assumption that different solutions of the integral equations (5.8) and (5.9) in the inner region 'prematch' one and same solution in the outer one. A similar conclusion that the free-surface position in the outer regions is insensitive to the different solutions in the inner one has been pointed out by Bazhlekov \& Chesters (1996) in the $\mathrm{L}-\mathrm{F}-\mathrm{S}$ case (see also $§ 7.1$ ).

\section{Comparisons with existing results}

\subsection{Liquid-fluid-solid case}

In the case of the $\mathrm{L}-\mathrm{F}-\mathrm{S}$ contact line a number of comparisons with the experimental results of Hoffman (1975) and Hansen \& Toong (1971) as well as with the numerical ones of Lowndes (1980) in the case of an advancing liquid-gas interface in a glass capillary tube at low Reynolds numbers are performed. The mathematical formulation, numerical technique and computational domain and finite-element meshes used in the present study resemble those used by Lowndes (1980), and so they are not described here. The two mathematical models differ from each other only in the slip condition: in the present study the slip condition (5.15) is used, while Lowndes has used a Navier slip condition (his equation (2.5)).

It is easy to see that the equation corresponding to (5.13) written in cylindrical coordinates can be obtained from Lowndes' equations (4.5) and (4.6). For the numerical results presented below the dimensionless slip length $\beta$ which appears in (5.15) is chosen to be equal to the slip coefficient in the Navier slip condition used by Lowndes, i.e. $\beta=10^{-6}$. In order to approximate the slip condition (5.15) well enough the dimensionless spatial step in a contact-line vicinity must be much smaller in magnitude than the slip length, $h_{\text {min }} \ll \beta$. The minimal dimensionless spatial step used in the present section as well as by Lowndes (1980) is of order $10^{-7}-10^{-9}$.

In figure 4 the results of the present study for the inclination of the meniscus $\theta$ are compared with the numerical results of Lowndes (1980) at different capillary numbers $\mathrm{Ca}$ and static contact angles $\theta_{s}$. 

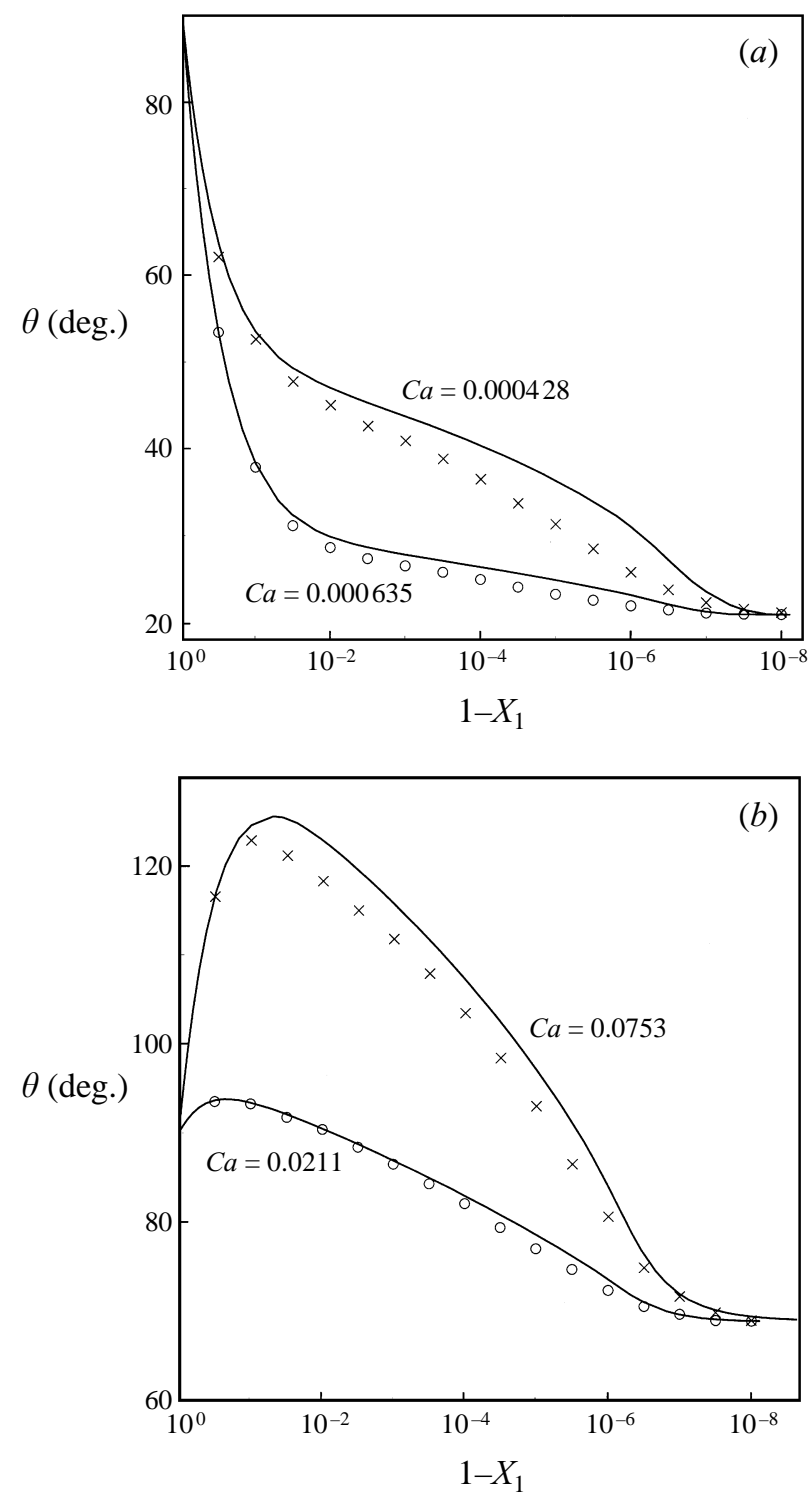

FIGURE 4. Comparison between the numerical results of the present paper (thick lines) and those of Lowndes $(\times, \bigcirc)$ at different capillary numbers and static contact angles: $(a) \theta_{s}=21^{\circ}$ and $(b)$ $\theta_{s}=69$.

\subsection{Liquid-fluid-liquid case}

In the case of the $\mathrm{L}-\mathrm{F}-\mathrm{L}$ contact line a number of comparisons with the experimental results of Torza \& Mason (1970) and Mori (1978) are presented. In Torza \& Mason (1970) the interaction between three immiscible liquids with practically equal densities has been investigated. They have studied the engulfing of one liquid drop by another, using shear and/or electrical fields to bring the drops together.

The numerical results are obtained without taking into account the external forces (gravity, electrical and shear fields). The shape shown on the first frame of figures 5 and 6 and zero velocity field were chosen as the initial ones. The characteristic length $l$ is equal to the equivalent radius of one of the drops (the volumes of both drops are 
(a)
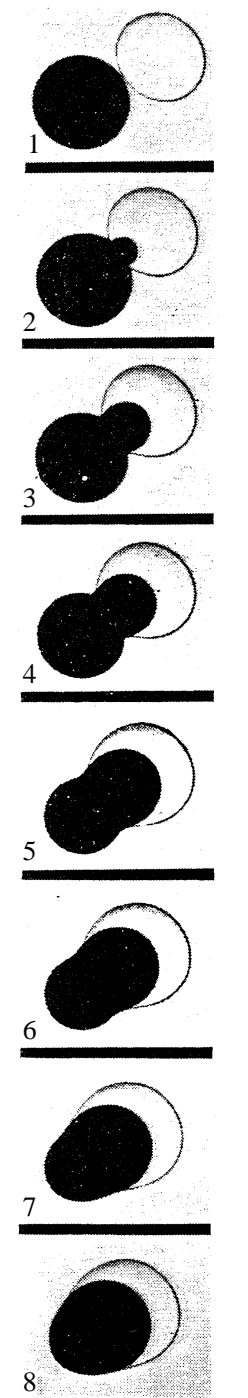

(b)
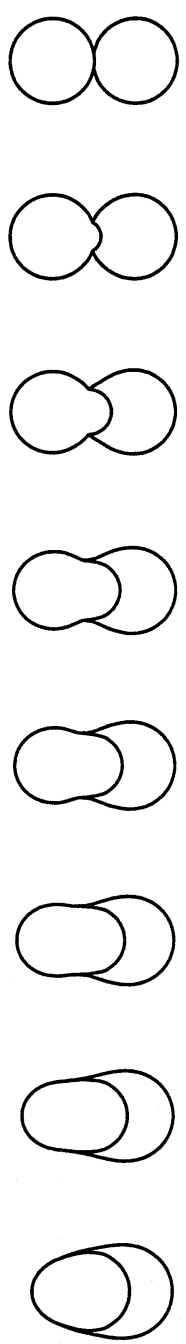

(c)
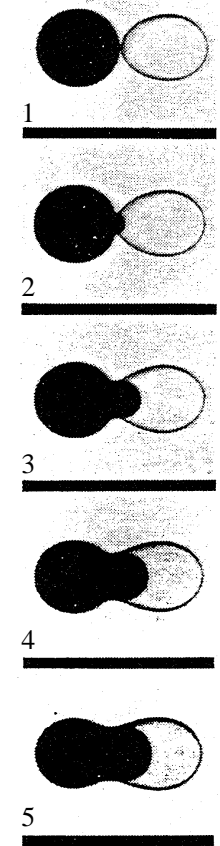

5
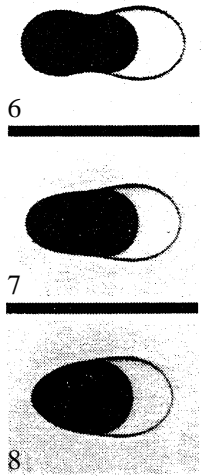

Figure 5. Comparison between the numerical $(b)$ and experimental $(a, c)$ results of Torza \& Mason (1970) concerning engulfing of one liquid drop by another. To bring the liquid drops together Torza $\&$ Mason have used a $(a)$ linear shear flow, and $(b)$ an electrical field.

assumed equal), and the characteristic time is $T=1 \mathrm{~s}$. The ambient liquid occupies the domain $\Omega_{1}$; the left part of the compound drop is $\Omega_{2}$, and the right part is $\Omega_{3}$.

In figure 5 a comparison between the numerical and experimental results is shown for the engulfing of a water drop by a Ucon oil LB-285 drop in silicone oil: $R e=0.012$, $W e=0.02, \lambda_{2}=\lambda_{3}=1, \eta_{2}=0.002, \eta_{3}=0.023, \kappa_{13}=0.115, \kappa_{23}=0.29 ; e=1$. The time between consecutive experimental frames is about $1 \mathrm{~s}$. The difference between the numerical and actual time is less than $15 \%$.

In figure 6 an analogous comparison is presented in the case when the second (righthand) part of the compound drop is Ucon oil 50-HB-55 (both of the other liquids are the same). In this case the surface tension coefficient $\sigma_{23}$ at the free interface between both parts of the compound drop is zero (i.e. the compound drop consists of two 
(a)
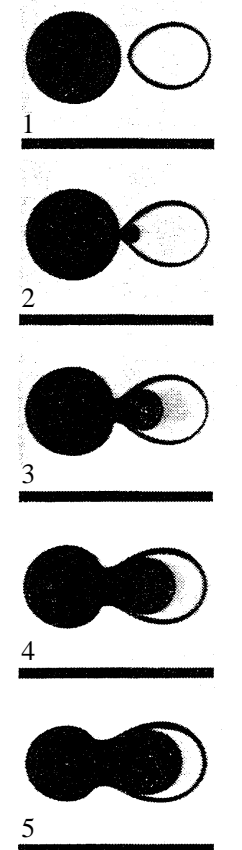

5

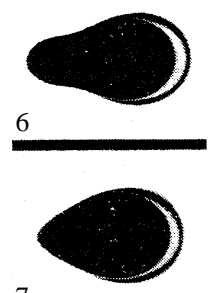

7 (b)
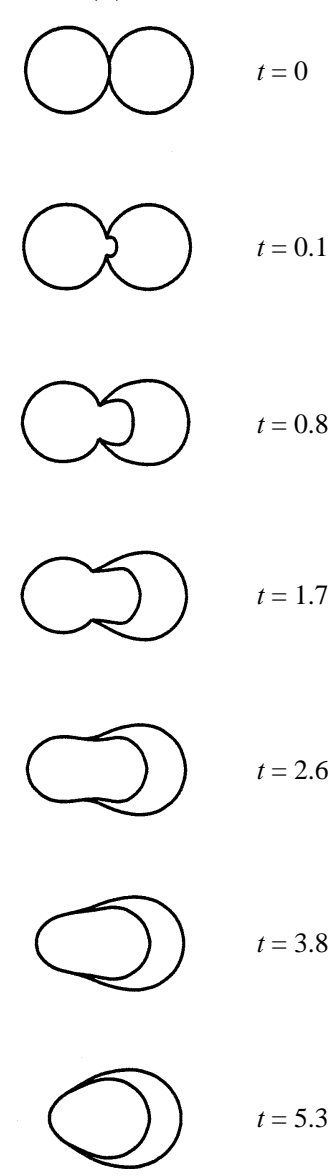

FIgURE 6. Comparison between the numerical $(b)$ and experimental $(a)$ results of Torza \& Mason (1970). The time (in s) shown on the figure corresponds to the numerical results.

miscible liquids). Also $R e=0.012, W e=0.02, \lambda_{2}=\lambda_{3}=1, \eta_{2}=0.002, \eta_{3}=0.003$, $\kappa_{13}=0.47, \kappa_{23}=0.0$, and $e=1$. To bring the single drops together Torza \& Mason (1970) applied an electrical field. In this case the differences between the numerical and experimental time is about $10 \%$.

In spite of the differences between the initial shapes and velocities and the applied external forces good qualitative agreement between the numerical and experimental results is obtained with respect to the shape evolution of the compound drop. For both cases presented in figures 5 and 6 the surface tension coefficient $\sigma_{12}$ is greater than sum of the other two $\left(\sigma_{12}>\sigma_{13}+\sigma_{23}\right)$, i.e. there is no static stage of the $3 \mathrm{~S}$ compound drop. In correspondence with this, at the end of the process the $3 \mathrm{~S}$ compound drop turns into a type-A one (i.e. the left-hand drop is completely covered by the right-hand one). The formation of the type-A compound drop has been observed by Torza \& Mason (see their figure $10 a, b, e)$, but these frames are not shown in figures 5 and 6 .

To conclude the present section another group of comparisons, with the experimental results of Mori (1978), are presented. Mori has studied the unsteady free rise of two-phase gas-liquid compound drops through another immiscible liquid in the 
(c)

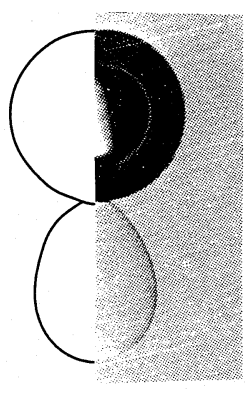

(b)

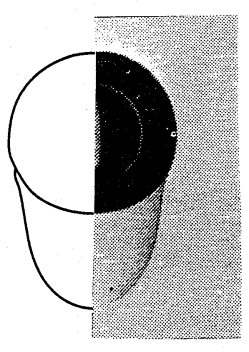

(a)

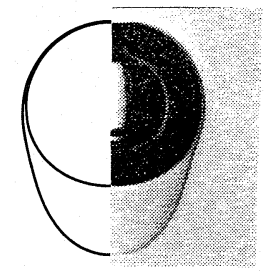

FIGURE 7. The unsteady free rise of a gas-liquid (aqueous glycerol) compound drop in castor oil: (a) (the earliest) corresponds to A-type configuration of the compound drop (without contact line).

gravitational field. The characteristic length and velocity, as well as the other notation, are the same as in the numerical test in $\S 6$. The upper phase of the compound drop $\left(\Omega_{3}\right)$ is gas.

Mori's experimental results on the unsteady free rise of a gas-liquid (aqueous glycerol) compound drop through castor oil are compared in figure 7, at $R e=0.009$, $W e=0.0024, \lambda_{2}=1.29, \eta_{2}=0.84, \kappa_{13}=2.57, \kappa_{23}=3.64$, and $e=0.52$, with the numerical results for the compound drop shape only. In other words the numerically obtained compound drop shapes presented on this figure are those which correspond best to the experimental ones. In practice two unsteady problems are solved. The first is the free rise of a type-A compound drop (figure 7(a) corresponds to a type-A compound drop). In this case the initial conditions are a perfectly symmetric compound drop shape (both interfaces are concentric spheres) and zero velocity field at $t=0$. At the end of the free rise of the type-A compound drop a thin film of the shell liquid has formed at its upper part. The second is the free rise of a $3 \mathrm{~S}$ compound drop. In this case the initial shape and velocity field are taken as those obtained at the end of the type-A compound drop rise. In other words part of the thin liquid film is replaced with a free interface $\left(\Gamma_{13}\right)$ between the ambient liquid and the inner phase. Thus, figures $7(b)$ and $7(c)$ correspond to the free rise of the $3 \mathrm{~S}$ compound drop. The tendency of separation of the two-phase compound drop which has been observed by Mori is also predicted by the numerical results. 
(a)

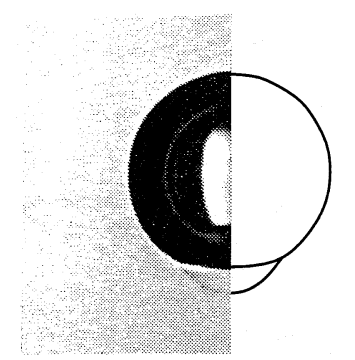

(b)

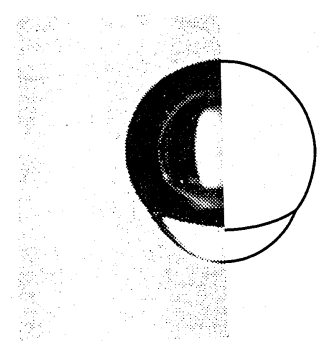

FIGURE 8. Comparison between the numerical (right) and experimental (left) steady shapes of $3 \mathrm{~S}$ gas-liquid compound drops: (a) castor oil in 96\% aqueous glycerol, $R e=0.048, W e=0.01 ; \lambda_{2}=$ $0.76, \eta_{2}=1.185, \kappa_{13}=3.64, \kappa_{23}=2.57, e=0.026$. (b) R113 in $86 \%$ aqueous glycerol, $R e=0.48$, $W e=0.06, \lambda=1.3, \eta=0.007 ; \kappa=1.94, \kappa=0.57, e=0.095$.

Figures $8(a)$ and $8(b)$ show comparisons between numerical and experimental (Mori 1978) steady shapes of a $3 \mathrm{~S}$ compound drop. These are the most objective comparisons presented here, because the steady shape of the compound drop does not depend on the initial conditions.

It is easy to see that in the last three comparisons considered here (figures 7 and 8) one of the surface tension coefficients is greater than the sum of the other two (in the first case, figure 7, $\sigma_{23}>\sigma_{13}+\sigma_{12}$, and in the other two cases, figure 8, $\sigma_{13}>\sigma_{12}+\sigma_{23}$ ), i.e. there are no static $3 \mathrm{~S}$ compound drop states, but a dynamic contact line exists and in some cases (see figure 7) the contact angles can take any value in the interval $[0, \pi]$.

\section{Conclusions}

The aim of the study is to check the possibility of numerical simulation of dynamic contact-line problems based on matching integral equations in a contact-line vicinity with the classical differential equations far from the contact line. This approach does not assume that the actual dynamic contact angles are equal to the static ones, which is the assumption in most of the theoretical studies.

The comparisons presented here between the numerical results obtained for some dynamic contact-line problems and the corresponding experimental and theoretical ones show that the approach considered here provides an acceptable basis for numerical simulation of those problems, even in the cases when the static contact angles are not defined or some of them are zero. The numerical technique presented, unfortunately, cannot provide predictions for the position of the free surfaces very close to the contact line, but only in the outer region. The reason for this probably is that an insufficiently adequate model is used in the inner region, where the influence of long-range intermolecular forces, precursor film (see Hocking 1993 and de Gennes 1985) or a molecular model could also be considered.

The authors gratefully acknowledge the financial assistance of the Commission of the European Community and the scientific support of Professor A. K. Chesters of the Eindhoven University of Technology (Netherlands), which enabled the generation of the $\mathrm{L}-\mathrm{F}-\mathrm{S}$ predictions within the framework of research project 8144 of S\&T Cooperation with Eastern and Central European Countries. The authors also thank John Muirhead for editorial help. This study was partially supported by Grant MM $501 / 95$ of the Bulgarian Ministry of Science and Technology. 


\section{REFERENCES}

Batchelor, G. K. 1967 An Introduction to Fluid Dynamics. Cambridge University Press.

BAzhlekov, I. B. \& Chesters, A. K. 1996 Numerical investigation of the dynamic influence of the contact line region on the macroscopic meniscus shape. J. Fluid Mech. 329, 137.

Bazhlekov, I. B. \& Shopov, P. J. 1990 Mathematical and numerical modeling of hydrodynamic problems with dynamic contact lines. C. R. Acad. Bulg. Sci. 43, 37.

Boender, W., Chesters, A. K. \& Zanden, A. J. J. van der 1991 An approximate analytical solution of the hydrodynamic problem associated with an advancing liquid-gas contact line. Intl J. Multiphase Flow 17, 661.

Christodoulou, K. N. \& Scriven, L. E. 1989 The fluid mechanics of slide coating. J. Fluid Mech. $208,321$.

Dussan V., E. B. 1976 The moving contact line: the slip boundary condition. J. Fluid Mech. 77, 665.

Dussan V., E. B. 1979 On the spreading of liquids on solid surfaces: static and dynamic contact lines. Ann. Rev. Fluid Mech. 11, 371.

Dussan V., E. B. \& Davis, S. H. 1974 On the motion of a fluid-fluid interface along a solid surface. J. Fluid Mech. 63, 71.

Dussan V., E. B., RAME, E. \& GAROFF, S. 1991 On identifying the appropriate boundary conditions at a moving contact line: an experimental investigation. J. Fluid Mech. 230, 97.

Gennes, P. G. DE 1985 Wetting: statics and dynamics. Rev. Mod. Phys. 57, 827.

HANSEN, R. J. \& ToONG, T. Y. 1971 Interface behavior as one fluid completely displaces another from a small-diameter tube. J. Colloid Interface Sci. 36, 410.

Hocking, L. M. 1977 A moving fluid interface. Part 2. The removal of the force singularity by a slip flow. J. Fluid Mech. 79, 209.

Hocking, L. M. 1992 Rival contact-angle models and the spreading drops. J. Fluid Mech. 239, 671.

Hocking, L. M. 1993 The influence of intermolecular forces on thin fluid layer. Phys. Fluids A 5, 793.

Hocking, L. M. \& Rivers, A. D. 1982 The spreading of a drop by capillary action. J. Fluid Mech. $121,425$.

Hoffman, R. 1975 A steady of the advancing interface. I. Interface shape in liquid-gas systems. $J$. Colloid Interface Sci. 50, 228.

HuH, C. \& MAson, S. G. 1977 The steady movement of a liquid meniscus in a capillary tube. J. Fluid Mech. 81, 401.

Ivanov, I. B., Kralchevsky, P. E. \& Nikolov, A. D. 1986 Film and line tension effects on the attachment of particles to an interface. J. Colloid Interface Sci. 112, 97.

JoHNSON, R. E. \& SADHAL, S. S. 1983 Stokes flow past bubbles and drops partially coated with thin films. Part 2. Thin films with internal circulation - a perturbation solutions. J. Fluid Mech. 132, 295.

Johnson, R. E. \& SAdHaL, S. S. 1985 Fluid dynamics of compound multiphase drops and bubbles. Ann. Rev. Fluid Mech. 17, 289.

KafKA, F. Y. \& Dussan, V., E. B. 1979 On the interpretation of dynamic contact angles in capillaries. J. Fluid Mech. 95, 539.

LownDEs, J. 1980 The numerical simulation of the steady motion of the fluid meniscus in a capillary tube. J. Fluid Mech. 101, 631.

MorI, Y. H. 1978 Configurations of gas-liquid two-phase bubbles in immiscible liquid media. Intl J. Multiphase Flow 4, 383.

NGan, C. G. \& Dussan V., E. B. 1982 On the nature of the dynamic contact angle: an experimental study. J. Fluid Mech. 118, 27.

NGan, C. G. \& Dussan V., E. B. 1989 On the dynamics of the liquid spreading on solid surfaces. J. Fluid Mech. 209, 191.

Nigmatulin, N. 1978 Mechanics of Heterogenous Mediums. Nauka, Moscow (in Russian).

PethicA, B. A. 1977 The contact angle equilibrium (letter to the editors). J. Colloid Interface Sci. 62, 567.

Shopov, P. J. \& BAZHLEKov, I. B. 1991 Numerical method for viscous hydrodynamic problems with dynamic contact lines. Comput. Methods Appl. Mech. Engng 91, 1157. 
Shopov, P. J., Minev, P. D. \& Bazhlekov, I. B. 1992 Numerical method for unsteady viscous hydrodynamical problem with free boundaries. Intl J. Numer. Meth. Fluids 14, 681.

Silliman, W. J. \& Skriven, L. E. 1980 Separating flow near a static contact line: slip at a wall and shape of a free surface. J. Comput. Phys. 34, 287.

Sullivan, D. E. \& Telo DA Gama, M. M. 1986 Wetting transition and multilayer adsorption at fluid interfaces. In Fluid Interfacial Phenomena (ed. C. A. Croxston), p. 45. Wiley \& Sons.

Torza, S. \& Mason, S. G. 1970 Three-phase interactions in shear and electrical fields. J. Colloid Interface Sci. 33, 67.

VuONG, S. T. \& SADHAL, S. S. 1989 Growth and translation of a liquid-vapor compound drop in a second liquid. Part 1. Fluid mechanics. J. Fluid Mech. 209, 617. 